\title{
ACE-inhibitory Effect and Physicochemical Characteristics of Yogurt Beverage Fortified with Whey Protein Hydrolysates
}

\author{
Sung-Min Lim, Na-Kyoung Lee, Keun-Kyu Park, Yoh Chang Yoon, and Hyun-Dong Paik* \\ Division of Animal Life Science and Bio/Molecular Informatics Center, Konkuk University, Seoul 143-701, Korea
}

\begin{abstract}
This study investigated the ACE-inhibitory effect of yogurt beverage fortified with hydrolysates as well as the suitability of hydrolysates as a nutraceutical additive to yogurt beverage. Three whey protein hydrolysates hydrolyzed by alcalase, protamex, and trypsin were each added to yogurt beverage at concentrations of $1.25,2.5$, and $5 \mathrm{mg} / \mathrm{mL}$. Yogurt beverage fortified with $2.5 \mathrm{mg} / \mathrm{mL}$ of hydrolysates had 61-69\% ACE-inhibitory activity, whereas yogurt beverage fortified with $5 \mathrm{mg} /$ $\mathrm{mL}$ of hydrolysates showed $74 \%$ ACE-inhibitory activity. There were no significant differences in ACE-inhibitory activity between the alcalase or protamex hydrolysates during storage; however, trypsin hydrolysate exhibited significant differences. On the other hand, physicochemical characteristics such as $\mathrm{pH}$ (3.47-3.77), titratable acidity (0.81-0.84\%), colority, viable cell count, and sensory qualities were not significantly different among the tested yogurt beverage samples during storage. These results showed that yogurt beverage fortified with whey protein hydrolysates maintained antihypertensive activity and underwent no unfavorable changes in physicochemical characteristics regardless of enzyme type.
\end{abstract}

Key words: angiotensin-converting enzyme, whey protein hydrolysate, yogurt beverage

\section{Introduction}

Angiotensin-converting enzyme (ACE) is a transmembrane zinc metallopeptidase that cleaves dipeptides from the $\mathrm{C}$-terminus of a peptide substrate. ACE is a regulatory factor in the renin-angiotensin system (RAS), which plays an important role in cardiovascular homeostasis, blood pressure regulation, and fluid and salt balance. In the RAS, rennin cleaves the liver-derived precursor angiotensinogen into a decapeptide, angiotensin I, which is then cleaved by ACE to form angiotensin II (a vasoconstrictor). In addition, ACE inactivates the vasodilative peptides bradykinin and kallidin and stimulates the release of aldosterone, which increases blood pressure (Campbell, 2003).

Several lactic acid bacteria are known to produce inhibitors of ACE during fermentation. These inhibitors are formed by proteinase in lactic acid bacteria during the hydrolysis of milk proteins, mainly casein, into peptides, which can then be used as nitrogen sources for bacterial growth. The total activity and specificity of proteolysis

\footnotetext{
*Corresponding author: Hyun-Dong Paik, Division of Animal Life Science and Bio/Molecular Informatics Center, Konkuk University, Seoul 143-701, Korea. Tel: 82-2-2049-6011, Fax: 82-2-455-3082, E-mail: hdpaik@konkuk.ac.kr
}

plays an important role in this process since the resulting inhibitors are peptides (FitzGerald and Murray, 2006; Korhonen and Pihlanto, 2006; López-Fandino et al., 2006). Some of these inhibitor peptides have also been shown to lower blood pressure in hypertensive rats (Fuglsang et al., 2002).

Peptides with ACE-inhibitory activity have already been isolated from various food proteins (Ariyoshi, 1993; Jang and Lee, 2005; Yamamoto et al., 1994). Especially, fermented milk products are known as an excellent source of bioactive peptides (Yamamoto, 1997). Such peptides possess antibacterial, anticancer, immunomodulatory, mineral-binding, and antihypertensive properties (Minervini et al., 2003; Shah, 2000; Silvia and Malcata, 2005). Extracellular proteinases are involved in the initial degradation of caseins, yielding a large number of oligopeptides. Consequently, further breakdown by intracellular peptidases is critical to making available essential and growth-stimulating amino acids and peptides (Christensen et al., 1999; Donkor et al., 2007; Kunji et al., 1996). Bioactive peptide fragments originating from whey proteins should be taken into account as components that have a positive effect on cardiovascular health (Clare and Swaisgood, 2000; Pihlanto-Leppala et al., 2000; Seppo et al., 2003; Yusuf et al., 1999). Bovine milk 
whey is considered one of the main raw materials in the production of functional ingredients. Several authors reported that whey protein hydrolysates show ACE-inhibitory activity as well as hypotensive activity in animals and humans (Fujita et al., 2001; Van der Ven et al., 2002; Vermeirssen et al., 2003).

The purpose of this study was to investigate the ACEinhibitory effect of yogurt beverage fortified with hydrolysates as well as whether or not hydrolysates are suitable as a nutraceutical additive to yogurt beverage. The results would determine the potential of whey protein hydrolysate as an ingredient for the production of advanced antihypertensive functional dairy foods.

\section{Materials and Methods}

\section{Enzymatic hydrolysis of whey protein concentrate}

Whey protein concentrate (WPC, 70\% proteins) was supplied by Hilmar Cheese Company (USA). The enzymes used in this study were alcalase, protamex, and trypsin (Novo Nordisk Biochem. Inc., Denmark). WPC was solubilized in deionized water $(10 \%(\mathrm{w} / \mathrm{w})$ protein basis). The enzyme preparation was then added to the homogenate at concentration of $1.25 \%(\mathrm{w} / \mathrm{v})$. The optimized hydrolysis conditions used for these enzymes were as follows: alcalase $\left(50^{\circ} \mathrm{C}, \mathrm{pH} 6.5,3 \mathrm{~h}\right)$, protamex $\left(50^{\circ} \mathrm{C}, \mathrm{pH}\right.$ $7.0,5 \mathrm{~h})$, and trypsin $\left(37^{\circ} \mathrm{C}, \mathrm{pH} 8.0,2 \mathrm{~h}\right)$. Hydrolysis was performed in a shaking incubator at $180 \mathrm{rpm}$ (Kim et al., 2007; Nicholas and Eric, 1996; Sinha et al., 2007).

After the reactions, the whey protein hydrolysates were placed in a water bath $\left(20 \mathrm{~min}, 120^{\circ} \mathrm{C}\right)$ for enzyme inactivation followed by cooling. The hydrolysates were centrifuged at 5,200 $\times \mathrm{g}$ for $15 \mathrm{~min}$, after which the supernatant was collected and frozen at $-70^{\circ} \mathrm{C}$ for $24 \mathrm{~h}$ before freezedrying in a chemical-free freeze drier (FDCF-12012, Operon, Korea).

\section{Sample preparation}

All yogurt beverage (Namyang yogurt beverage, Namyang Dairy Products Co., Korea) samples were obtained from a local shop in Seoul. The hydrolysates were added at concentrations of $1.25,2.5$, and $5 \mathrm{mg} / \mathrm{mL}$ of yogurt beverage. For storage tests, the samples were placed in a $4^{\circ} \mathrm{C}$ refrigerator for up to $7 \mathrm{~d}$. The day after the designated expiration date was fixed as $0 \mathrm{~d}$ of storage, and samples were taken at 1 or $2 \mathrm{~d}$ intervals for analytical and microbial testing.

\section{In vitro assay for $\mathrm{ACE}$ inhibitory activity}

ACE-inhibitory activity was measured using a modified method (Cushman and Cheung, 1971; Van der Ven et al., 2002; Vermeirssen et al., 2003). For each assay, a mixture containing $50 \mu \mathrm{L}$ of sample, $100 \mu \mathrm{L}$ of $0.1 \mathrm{M}$ sodium

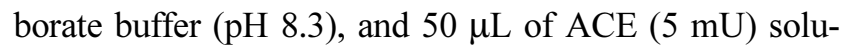
tion was incubated at $37^{\circ} \mathrm{C}$ for $5 \mathrm{~min}$. Then, $50 \mu \mathrm{L}$ of Hip-His-Leu solution was added and incubated for 30 min. The enzymatic reaction was stopped by adding 250 $\mu \mathrm{L}$ of $0.5 \mathrm{~N} \mathrm{HCl}$. Hippuric acid forced by the action of the ACE on HHL was extracted from the acidified solution into $1.5 \mathrm{~mL}$ of ethyl acetate by vortexing for $15 \mathrm{~s}$. Then, centrifugation was carried out at 3,290 $\mathrm{g}$ for $10 \mathrm{~min}$ at $4^{\circ} \mathrm{C}$, after which a $1 \mathrm{~mL}$ aliquot of each ethyl acetate layer was transferred into a clean tube and evaporated by heating at $90^{\circ} \mathrm{C}$ for $60 \mathrm{~min}$ in a water bath. The hippuric acid was redissolved in $3 \mathrm{~mL}$ of deionized water, and the amount formed was determined by its absorbance at 228 $\mathrm{nm}$. The activity of each sample was tested in triplicate. ACE- inhibitory activity was calculated according to the following formula:

$$
\begin{aligned}
& \text { ACE-inhibitory activity }(\%) \\
& =\frac{\text { absorbance of control-absorbance of sample }}{\text { absorbance of control }} \times 100
\end{aligned}
$$

\section{pH, titratable acidity, and color evaluation}

The $\mathrm{pH}$ levels of the samples were determined using a pH meter (Model 340, Mettler-Toledo GmbH, Schwerzenbach, Switzerland). The $\mathrm{pH}$ values of the yogurt beverage samples were each measured in $30 \mathrm{~mL}$ of sample. The titratable acidity was determined according to the Association of Official Analytical Chemists (AOAC, 1995) method.

Instrumental color analysis of yogurt was performed as follows. The yogurt beverage sample was measured (40 $\mathrm{mL})$ in Petri dishes $(90 \times 15 \mathrm{~mm})$ for color analysis, and color measurements were taken using a colorimeter (Chroma meter CR-400, Konica-Minolta, Japan), which contained an $8 \mathrm{~mm}$ diameter measuring area and a $50 \mathrm{~mm}$ diameter illumination area. Color values (CIE L*, a*, and $\left.b^{*}\right)$ were measured on the surfaces of the samples, and the results are in the mean of triplicate measurements.

\section{Microbiological analysis}

Viable cells were counted using the spread plate method with MRS agar. The plates were incubated at $35^{\circ} \mathrm{C}$ for $48 \mathrm{~h}$. 


\section{Sensory evaluation}

Sensory evaluation of yogurt samples was carried out at 0,3 , and $7 \mathrm{~d}$ using a five-point hedonic scale ranging from "dislike extremely" to "like extremely". Ten panelists ( 5 females, 5 males) were selected by screening tests conducted with dairy product based on interest, availability, and performance, and these who confirmed consuming yogurt at least once a week were chosen for this study.

\section{Statistical analysis}

All experiments were performed at least three times under each experimental condition and mean values were reported. Analysis of variance (ANOVA) was performed on all of the variables measured using the General Linear Model (GLM) procedure of the SAS statistical package (SAS Institute, Inc., 1999). Duncan's multiple range test $(p<0.05)$ was used to determine differences between treatment means.

\section{Results and Discussion}

\section{ACE inhibitory activity of yogurt fortified with hydrolysates}

Numerous studies have focused on the production and isolation of ACE-inhibitory materials (Fujita et al., 2001; Van der Ven et al., 2002; Vermeirssen et al., 2003). Fig. 1 shows that the ACE-inhibitory activity of yogurt beverage ranged from 47 to $50 \%$. Japanese Calpis yogurt reportedly has similar ACE-inhibitory activity, but it is dependent on the seed culture (Otte et al., 2003). Therefore, this study studied yogurt beverage fortified with WPH according to enzyme type. Yogurt beverage fortified with $1.25 \mathrm{mg} / \mathrm{mL}$ of protamex hydrolysates possessed approximately 57-64\% ACE-inhibitory activity. Yogurt beverage fortified with $2.5 \mathrm{mg} / \mathrm{mL}$ of alcalase hydrolysates had approximately 61-69\% ACE-inhibitory activity. Finally, yogurt beverage fortified with $2.5 \mathrm{mg} / \mathrm{mL}$ of trypsin hydrolysates had similar ACE-inhibitory activity. Yogurt beverage fortified with $5 \mathrm{mg} / \mathrm{mL}$ of protamex hydrolysates had a maximum ACE-inhibitory activity of $74 \%$. Yogurt beverage fortified with hydrolysates did not significant during storage $\left(R^{2}=0.94\right)$.

\section{pH and titratable acidity}

The $\mathrm{pH}$ values for yogurt samples at $1 \mathrm{~d}$ ranged from 3.53 to 3.73 (Table 1). No significant differences were noted between the samples during storage. Changes in titratable acidity were not significantly different among
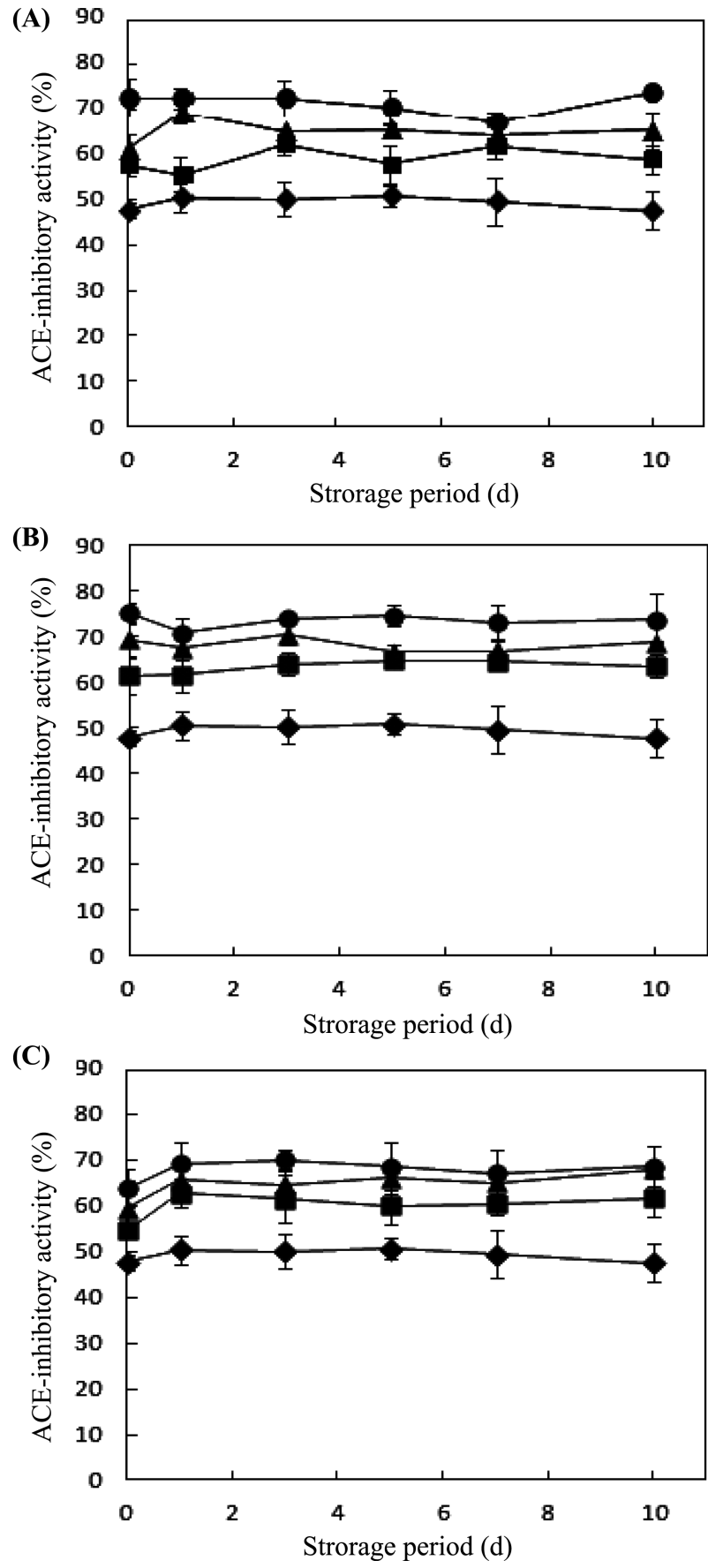

Fig. 1. Angiotensin-converting enzyme (ACE)-inhibitory activity of yogurt beverage fortified with whey protein hydrolysates. Hydrolysates concentrations were adjusted to $1.25 \mathrm{mg} / \mathrm{mL}(\boldsymbol{\square}), 2.5 \mathrm{mg} / \mathrm{mL}(\boldsymbol{\Delta}) 5.0 \mathrm{mg} / \mathrm{mL}(\boldsymbol{O})$, or 0 $\mathrm{mg} / \mathrm{mL}(\bullet)$. (A) alcalase, (B) protamex, and (C) trypsin

the hydrolysates during storage, and titratable acidity values ranged from $0.81 \%$ to $0.83 \%$ at $7 \mathrm{~d}$ (Table 2 ).

\section{Color evaluation}

In the color evaluation, $\mathrm{L}^{*}$ value (lightness) was not significantly different among the enzymatic hydrolysatetreated samples, whereas a* (redness) and $\mathrm{b}^{*}$ (yellowness) 
Table 1. Changes in pH value of yogurt beverage fortified with whey protein hydrolysates

\begin{tabular}{ccccccc}
\hline \hline \multirow{2}{*}{ Enzyme } & $\begin{array}{c}\text { Hydrolysate conc. } \\
(\mathrm{mg} / \mathrm{mL})\end{array}$ & 0 & 1 & 3 & 5 & 7 \\
\cline { 3 - 7 } & 0 & $3.63 \pm 0.02$ & $3.53 \pm 0.02$ & $3.55 \pm 0.01$ & $3.54 \pm 0.02$ & $3.64 \pm 0.01$ \\
\hline Control & 1.25 & $3.64 \pm 0.03$ & $3.63 \pm 0.01$ & $3.53 \pm 0.07$ & $3.47 \pm 0.12$ & $3.63 \pm 0.07$ \\
Alcalase & 2.5 & $3.61 \pm 0.02$ & $3.67 \pm 0.02$ & $3.56 \pm 0.04$ & $3.53 \pm 0.07$ & $3.66 \pm 0.05$ \\
& 5.0 & $3.63 \pm 0.01$ & $3.69 \pm 0.07$ & $3.65 \pm 0.03$ & $3.63 \pm 0.01$ & $3.77 \pm 0.02$ \\
\hline \multirow{3}{*}{ Protamex } & 1.25 & $3.49 \pm 0.09$ & $3.63 \pm 0.02$ & $3.50 \pm 0.13$ & $3.59 \pm 0.04$ & $3.66 \pm 0.02$ \\
& 2.5 & $3.57 \pm 0.01$ & $3.65 \pm 0.05$ & $3.57 \pm 0.04$ & $3.60 \pm 0.02$ & $3.70 \pm 0.02$ \\
& 5.0 & $3.60 \pm 0.02$ & $3.73 \pm 0.02$ & $3.67 \pm 0.02$ & $3.62 \pm 0.03$ & $3.76 \pm 0.01$ \\
\hline \multirow{3}{*}{ Trypsin } & 1.25 & $3.53 \pm 0.01$ & $3.63 \pm 0.03$ & $3.57 \pm 0.01$ & $3.61 \pm 0.05$ & $3.66 \pm 0.03$ \\
& 2.5 & $3.55 \pm 0.03$ & $3.68 \pm 0.02$ & $3.61 \pm 0.01$ & $3.60 \pm 0.02$ & $3.72 \pm 0.01$ \\
\hline
\end{tabular}

All values are the mean $\pm \mathrm{SD}$ of three replicates.

Table 2. Changes in titratable acidity of yogurt beverage fortified with whey protein hydrolysates

\begin{tabular}{ccccccc}
\hline \hline \multirow{2}{*}{ Enzyme } & \multirow{2}{*}{$\begin{array}{c}\text { Hydrolysate conc. } \\
(\mathrm{mg} / \mathrm{mL})\end{array}$} & 0 & \multicolumn{5}{c}{ Storage period (d) } \\
\cline { 3 - 7 } & 0 & $0.81 \pm 0.01$ & $0.81 \pm 0.01$ & $0.82 \pm 0.01$ & $0.82 \pm 0.01$ & $0.83 \pm 0.01$ \\
\hline Control & 1.25 & $0.81 \pm 0.01$ & $0.82 \pm 0.01$ & $0.83 \pm 0.01$ & $0.82 \pm 0.01$ & $0.82 \pm 0.01$ \\
Alcalase & 2.5 & $0.83 \pm 0.01$ & $0.82 \pm 0.01$ & $0.82 \pm 0.01$ & $0.83 \pm 0.01$ & $0.83 \pm 0.01$ \\
& 5.0 & $0.84 \pm 0.01$ & $0.81 \pm 0.01$ & $0.82 \pm 0.01$ & $0.83 \pm 0.01$ & $0.81 \pm 0.01$ \\
\hline \multirow{3}{*}{ Protamex } & 1.25 & $0.84 \pm 0.01$ & $0.83 \pm 0.01$ & $0.82 \pm 0.01$ & $0.82 \pm 0.01$ & $0.82 \pm 0.01$ \\
& 2.5 & $0.84 \pm 0.01$ & $0.81 \pm 0.01$ & $0.83 \pm 0.01$ & $0.81 \pm 0.01$ & $0.81 \pm 0.01$ \\
& 5.0 & $0.84 \pm 0.01$ & $0.82 \pm 0.01$ & $0.82 \pm 0.01$ & $0.82 \pm 0.01$ & $0.81 \pm 0.01$ \\
\hline \multirow{3}{*}{ Trypsin } & 1.25 & $0.81 \pm 0.01$ & $0.81 \pm 0.01$ & $0.81 \pm 0.01$ & $0.81 \pm 0.01$ & $0.81 \pm 0.01$ \\
& 2.5 & $0.82 \pm 0.01$ & $0.81 \pm 0.01$ & $0.81 \pm 0.01$ & $0.82 \pm 0.01$ & $0.82 \pm 0.01$ \\
& 5.0 & $0.82 \pm 0.01$ & $0.81 \pm 0.01$ & $0.82 \pm 0.01$ & $0.82 \pm 0.01$ & $0.82 \pm 0.01$ \\
\hline
\end{tabular}

All values are the mean $\pm \mathrm{SD}$ of three replicates.

Table 3. Changes in color values of yogurt beverage fortified with whey protein hydrolysates

\begin{tabular}{|c|c|c|c|c|c|c|c|c|c|c|}
\hline \multirow{2}{*}{$\begin{array}{c}\text { Storage } \\
\text { period }(\mathrm{d})\end{array}$} & \multirow{2}{*}{ Control } & \multicolumn{3}{|c|}{ Alcalase hydrolysate $(\mathrm{mg} / \mathrm{mL})$} & \multicolumn{3}{|c|}{ Protamex hydrolysate $(\mathrm{mg} / \mathrm{mL})$} & \multicolumn{3}{|c|}{ Trypsin hydrolysate (mg/mL) } \\
\hline & & 1.25 & 2.5 & 5.0 & 1.25 & 2.5 & 5.0 & 1.25 & 2.5 & 5.0 \\
\hline CIE L* & $66.21 \pm 0.01$ & $66.55 \pm 0.11$ & $66.13 \pm 0.01$ & $66.58 \pm 0.02$ & $65.81 \pm 0.20$ & $66.24 \pm 0.02$ & $66.19 \pm 0.13$ & $66.46 \pm 0.02$ & $66.50 \pm 0.05$ & $66.97 \pm 0.13$ \\
\hline \multirow{2}{*}{$\begin{array}{l}\text { CIE } a^{*} \\
\text { CIE } b^{*}\end{array}$} & $1.22 \pm 0.01$ & $0.74 \pm 0.09$ & $1.42 \pm 0.01$ & $0.83 \pm 0.03$ & $1.37 \pm 0.04$ & $1.18 \pm 0.01$ & $0.95 \pm 0.03$ & $1.30 \pm 0.02$ & $1.18 \pm 0.01$ & $1.61 \pm 0.06$ \\
\hline & $23.66 \pm 0.02$ & $22.34 \pm 0.17$ & $23.69 \pm 0.03$ & $22.65 \pm 0.06$ & $23.53 \pm 0.23$ & $23.59 \pm 0.02$ & $22.52 \pm 0.01$ & $23.77 \pm 0.03$ & $23.76 \pm 0.08$ & $24.30 \pm 0.19$ \\
\hline \multirow{2}{*}{$\begin{array}{l}\text { CIE L* } \\
\text { CIE a* }\end{array}$} & 0.04 & $65 \pm 0.52$ & $66.22 \pm 0.01$ & $.50 \pm 0.02$ & $65.96 \pm 0.01$ & $66.20 \pm 0.02$ & $66.42 \pm 0.02$ & $66.33 \pm 0.02$ & 0.06 & $66.69 \pm 0.02$ \\
\hline & $1.19 \pm 0.03$ & $0.50 \pm 0.06$ & $1.53 \pm 0.01$ & $0.83 \pm 0.03$ & $1.53 \pm 0.03$ & $1.17 \pm 0.01$ & $1.06 \pm 0.01$ & $1.16 \pm 0.01$ & $1.30 \pm 0.10$ & $1.39 \pm 0.01$ \\
\hline CIE b* & $23.82 \pm 0.19$ & $19.28 \pm 0.63$ & $24.01 \pm 0.01$ & $22.88 \pm 0.01$ & $23.92 \pm 0.01$ & $23.76 \pm 0.01$ & $23.39 \pm 0.02$ & $23.81 \pm 0.02$ & $23.82 \pm 0.30$ & $23.96 \pm 0.01$ \\
\hline CIE L* & $66.48 \pm 0.03$ & $66.80 \pm 0.03$ & $66.48 \pm 0.02$ & $66.91 \pm 0.03$ & $66.43 \pm 0.02$ & $66.63 \pm 0.03$ & $66.91 \pm 0.02$ & $66.72 \pm 0.01$ & $66.86 \pm 0.01$ & $67.07 \pm 0.04$ \\
\hline CIE a* & $1.24 \pm 0.02$ & $0.71 \pm 0.05$ & $1.41 \pm 0.02$ & $0.94 \pm 0.04$ & $1.63 \pm 0.06$ & $1.36 \pm 0.03$ & $1.21 \pm 0.01$ & $1.20 \pm 0.01$ & $1.29 \pm 0.01$ & $1.52 \pm 0.04$ \\
\hline $\mathrm{CIE} b^{*}$ & $23.25 \pm 0.05$ & $22.00 \pm 0.03$ & $23.37 \pm 0.04$ & $22.43 \pm 0.04$ & $23.59 \pm 0.03$ & $23.45 \pm 0.05$ & $23.12 \pm 0.03$ & $23.32 \pm 0.01$ & $23.44 \pm 0.01$ & $23.59 \pm 0.04$ \\
\hline CIE L* & $66.22 \pm 0.01$ & $66.58 \pm 0.01$ & $66.37 \pm 0.03$ & $66.93 \pm 0.03$ & $66.16 \pm 0.04$ & $66.56 \pm 0.01$ & $66.68 \pm 0.08$ & $66.65 \pm 0.07$ & $06.89 \pm 0.11$ & $0 / .00 \pm 0.01$ \\
\hline CIE $a^{*}$ & $1.12 \pm 0.02$ & $0.64 \pm 0.03$ & $1.54 \pm 0.06$ & $1.06 \pm 0.01$ & $1.51 \pm 0.04$ & $1.48 \pm 0.01$ & $1.23 \pm 0.08$ & $1.38 \pm 0.03$ & $1.45 \pm 0.04$ & $1.68 \pm 0.01$ \\
\hline CIE b* & $23.09 \pm 0.01$ & $21.92 \pm 0.01$ & $23.41 \pm 0.04$ & $22.76 \pm 0.02$ & $23.41 \pm 0.06$ & $23.61 \pm 0.01$ & $23.11 \pm 0.10$ & $23.59 \pm 0.10$ & $23.73 \pm 0.16$ & $23.77 \pm 0.01$ \\
\hline CIE L* & $66.14 \pm 0.01$ & $66.45 \pm 0.02$ & $66.15 \pm 0.01$ & $66.62 \pm 0.01$ & $66.06 \pm 0.03$ & $66.26 \pm 0.01$ & $66.49 \pm 0.01$ & $66.35 \pm 0.02$ & $66.53 \pm 0.03$ & $66.70 \pm 0.01$ \\
\hline \multirow{2}{*}{$\begin{array}{l}7 \text { CIE a* } \\
\text { CIE b* }\end{array}$} & $1.18 \pm 0.01$ & $0.71 \pm 0.03$ & $1.49 \pm 0.01$ & $1.04 \pm 0.01$ & $1.60 \pm 0.01$ & $1.31 \pm 0.01$ & $1.18 \pm 0.01$ & $1.18 \pm 0.02$ & $1.31 \pm 0.04$ & $1.43 \pm 0.01$ \\
\hline & $22.83 \pm 0.02$ & $21.71 \pm 0.03$ & $22.99 \pm 0.02$ & $22.26 \pm 0.01$ & $23.16 \pm 0.04$ & $23.06 \pm 0.02$ & $22.75 \pm 0.02$ & $22.96 \pm 0.02$ & $23.08 \pm 0.02$ & $23.18 \pm 0.01$ \\
\hline
\end{tabular}

All values are the mean $\pm \mathrm{SD}$ of three replicates. 
values were higher than that of the control. However, the observed color changes were not significantly among the samples during storage (Table 3).

\section{Microbiological analysis}

Viable LAB was counted using the spread plate method with MRS agar. The plates were incubated at $35^{\circ} \mathrm{C}$ for 48 $\mathrm{h}$. The results imply that the physicochemical characteristics of the hydrolysates are quite adequate for inclusion in yogurt, as no unfavorable changes in yogurt beverage for- tified with hydrolysates were observed (Table 4).

\section{Sensory evaluation}

Sensory evaluation of the yogurt beverage samples was carried out at 0,3 , and $7 \mathrm{~d}$ using a five-point hedonic scale ranging from "dislike extremely" to "like extremely". Sensory evaluation was not significantly different at any storage point $(0,3$, and $7 \mathrm{~d})$ or any enzymatic hydrolysate concentration (Tables 5-7). However, the value of mouth feel of $0 \mathrm{~d}$ and color of $5.0 \mathrm{mg} / \mathrm{mL}$ was significant differ-

Table 4. Changes in viable cells $(\log \mathrm{CFU} / \mathrm{mL})$ in yogurt beverage fortified with whey protein hydrolysates

\begin{tabular}{|c|c|c|c|c|c|c|}
\hline \multirow{2}{*}{ Enzyme } & \multirow{2}{*}{$\begin{array}{l}\text { Hydrolysate conc. } \\
(\mathrm{mg} / \mathrm{mL})\end{array}$} & \multicolumn{5}{|c|}{ Storage period (d) } \\
\hline & & 0 & 1 & 3 & 5 & 7 \\
\hline Control & 0 & $8.64 \pm 0.04$ & $8.67 \pm 0.06$ & $8.68 \pm 0.02$ & $8.68 \pm 0.06$ & $8.72 \pm 0.06$ \\
\hline \multirow{3}{*}{ Alcalase } & 1.25 & $8.58 \pm 0.04$ & $8.68 \pm 0.12$ & $8.70 \pm 0.06$ & $8.67 \pm 0.02$ & $8.68 \pm 0.07$ \\
\hline & 2.5 & $8.64 \pm 0.13$ & $8.70 \pm 0.04$ & $8.60 \pm 0.02$ & $8.68 \pm 0.08$ & $8.66 \pm 0.03$ \\
\hline & 5.0 & $8.61 \pm 0.08$ & $8.57 \pm 0.06$ & $8.65 \pm 0.04$ & $8.73 \pm 0.10$ & $8.67 \pm 0.06$ \\
\hline \multirow{3}{*}{ Protamex } & 1.25 & $8.65 \pm 0.08$ & $8.62 \pm 0.04$ & $8.66 \pm 0.07$ & $8.70 \pm 0.08$ & $8.66 \pm 0.04$ \\
\hline & 2.5 & $8.65 \pm 0.11$ & $8.64 \pm 0.05$ & $8.62 \pm 0.04$ & $8.66 \pm 0.06$ & $8.65 \pm 0.01$ \\
\hline & 5.0 & $8.65 \pm 0.06$ & $8.64 \pm 0.04$ & $8.65 \pm 0.09$ & $8.66 \pm 0.05$ & $8.70 \pm 0.07$ \\
\hline \multirow{3}{*}{ Trypsin } & 1.25 & $8.62 \pm 0.06$ & $8.64 \pm 0.03$ & $8.63 \pm 0.08$ & $8.72 \pm 0.10$ & $8.73 \pm 0.04$ \\
\hline & 2.5 & $8.63 \pm 0.04$ & $8.61 \pm 0.05$ & $8.70 \pm 0.10$ & $8.65 \pm 0.10$ & $8.70 \pm 0.12$ \\
\hline & 5.0 & $8.62 \pm 0.03$ & $8.64 \pm 0.06$ & $8.69 \pm 0.07$ & $8.66 \pm 0.10$ & $8.67 \pm 0.08$ \\
\hline
\end{tabular}

All values are the mean \pm SD of three replicates.

Table 5. Changes in sensory scores of yogurt beverage fortified with alcalase hydrolysate

\begin{tabular}{cccccc}
\hline \hline \multirow{2}{*}{$\begin{array}{c}\text { Sensory } \\
\text { parameter }\end{array}$} & Storage & \multicolumn{4}{c}{ Hydrolysate conc. $(\mathrm{mg} / \mathrm{mL})$} \\
\cline { 3 - 6 } & & 0 & 1.25 & 2.5 & 5.0 \\
\hline \multirow{3}{*}{ Odor } & 0 & $4.1 \pm 0.32$ & $4.0 \pm 0.47$ & $4.3 \pm 0.48$ & $4.3 \pm 0.48$ \\
& 3 & $4.0 \pm 0.67$ & $4.0 \pm 0.67$ & $3.9 \pm 0.57$ & $3.9 \pm 0.57$ \\
& 7 & $4.2 \pm 0.63$ & $4.2 \pm 0.63$ & $4.2 \pm 0.63$ & $4.3 \pm 0.67$ \\
\hline \multirow{3}{*}{ Taste } & 0 & $4.1 \pm 0.32$ & $4.1 \pm 0.32$ & $4.3 \pm 0.48$ & $4.1 \pm 0.32$ \\
& 3 & $4.0 \pm 0.67$ & $3.9 \pm 0.57$ & $4.1 \pm 0.74$ & $4.0 \pm 0.67$ \\
& 7 & $4.3 \pm 0.67$ & $4.4 \pm 0.70$ & $4.3 \pm 0.67$ & $4.4 \pm 0.70$ \\
\hline \multirow{2}{*}{ Mouth } & 0 & $4.3 \pm 0.48^{\mathrm{ab}}$ & $4.1 \pm 0.32^{\mathrm{b}}$ & $4.2 \pm 0.42^{\mathrm{ab}}$ & $4.6 \pm 0.52^{\mathrm{Aa}}$ \\
feel & 3 & $3.9 \pm 0.57$ & $4.0 \pm 0.67$ & $4.0 \pm 0.67$ & $3.8 \pm 0.42^{\mathrm{B}}$ \\
& 7 & $4.1 \pm 0.57$ & $4.4 \pm 0.70$ & $4.5 \pm 0.71$ & $4.2 \pm 0.63^{\mathrm{AB}}$ \\
\hline \multirow{3}{*}{ Color } & 0 & $4.2 \pm 0.32$ & $4.2 \pm 0.42$ & $4.3 \pm 0.48$ & $4.5 \pm 0.53^{\mathrm{A}}$ \\
& 3 & $4.0 \pm 0.67$ & $4.0 \pm 0.67$ & $4.0 \pm 0.67$ & $3.9 \pm 0.57^{\mathrm{B}}$ \\
& 7 & $4.1 \pm 0.57$ & $4.5 \pm 0.71$ & $4.4 \pm 0.70$ & $4.2 \pm 0.63^{\mathrm{AB}}$ \\
\hline \multirow{3}{*}{ Total } & 0 & $4.1 \pm 0.57$ & $4.4 \pm 0.70$ & $4.2 \pm 0.42$ & $4.2 \pm 0.42$ \\
& 3 & $3.9 \pm 0.57$ & $4.0 \pm 0.67$ & $4.0 \pm 0.67$ & $3.8 \pm 0.42$ \\
& 7 & $4.2 \pm 0.63$ & $4.3 \pm 0.67$ & $4.5 \pm 0.71$ & $4.3 \pm 0.67$
\end{tabular}

All values are the mean \pm SD of three replicates.

Sensory scores were assessed on a 5 point scale based on $1=$ extremely bad, $5=$ extremely good.

${ }^{\mathrm{A}, \mathrm{B}}$ Means within columns with different superscripts are significantly different $(p<0.05)$.

${ }^{\mathrm{a}, \mathrm{b}}$ Means within row with different superscripts are significantly different $(p<0.05)$.
Table 6. Changes in sensory scores of yogurt beverage fortified with protamex hydrolysate

\begin{tabular}{cccccc}
\hline \hline \multirow{2}{*}{$\begin{array}{c}\text { Sensory } \\
\text { parameter }\end{array}$} & $\begin{array}{c}\text { Storage } \\
\text { period (d) }\end{array}$ & \multicolumn{4}{c}{ Hydrolysate conc. $(\mathrm{mg} / \mathrm{mL})$} \\
\cline { 3 - 6 } Odor & 0 & $4.1 \pm 0.32$ & $3.9 \pm 0.32^{\mathrm{B}}$ & $4.0 \pm 0.47^{\mathrm{B}}$ & $4.2 \pm 0.42$ \\
& 3 & $4.0 \pm 0.67$ & $4.2 \pm 0.63^{\mathrm{AB}}$ & $4.6 \pm 0.70^{\mathrm{A}}$ & $4.5 \pm 0.53$ \\
& 7 & $4.2 \pm 0.63$ & $4.4 \pm 0.52^{\mathrm{A}}$ & $4.4 \pm 0.52^{\mathrm{AB}}$ & $4.2 \pm 0.42$ \\
\hline \multirow{3}{*}{ Taste } & 0 & $4.1 \pm 0.32$ & $4.2 \pm 0.42$ & $4.1 \pm 0.32^{\mathrm{B}}$ & $4.1 \pm 0.32$ \\
& 3 & $4.0 \pm 0.67^{\mathrm{b}}$ & $4.1 \pm 0.57^{\mathrm{ab}}$ & $4.6 \pm 0.52^{\mathrm{Aa}}$ & $4.5 \pm 0.53^{\mathrm{ab}}$ \\
& 7 & $4.3 \pm 0.67$ & $4.3 \pm 0.48$ & $4.3 \pm 0.48^{\mathrm{AB}}$ & $4.2 \pm 0.42$ \\
\hline \multirow{2}{*}{ Mouth } & 0 & $4.3 \pm 0.48$ & $4.1 \pm 0.32$ & $4.2 \pm 0.42$ & $4.1 \pm 0.32$ \\
feel & 3 & $3.9 \pm 0.57^{\mathrm{b}}$ & $4.2 \pm 0.63^{\mathrm{ab}}$ & $4.6 \pm 0.52^{\mathrm{a}}$ & $4.4 \pm 0.52^{\mathrm{ab}}$ \\
& 7 & $4.1 \pm 0.57$ & $4.1 \pm 0.32$ & $4.2 \pm 0.42$ & $4.3 \pm 0.48$ \\
\hline \multirow{3}{*}{ Color } & 0 & $4.2 \pm 0.32$ & $4.1 \pm 0.32$ & $4.1 \pm 0.32$ & $4.1 \pm 0.32$ \\
& 3 & $4.0 \pm 0.67$ & $4.3 \pm 0.67$ & $4.4 \pm 0.52$ & $4.3 \pm 0.48$ \\
& 7 & $4.1 \pm 0.57$ & $4.2 \pm 0.42$ & $4.2 \pm 0.42$ & $4.2 \pm 0.42$ \\
\hline \multirow{2}{*}{ Total } & 0 & $4.1 \pm 0.57$ & $4.2 \pm 0.42$ & $4.1 \pm 0.32$ & $4.1 \pm 0.32$ \\
& 3 & $3.9 \pm 0.57$ & $4.3 \pm 0.67$ & $4.3 \pm 0.48$ & $4.4 \pm 0.52$ \\
& 7 & $4.2 \pm 0.63$ & $4.4 \pm 0.52$ & $4.3 \pm 0.48$ & $4.2 \pm 0.42$ \\
\hline
\end{tabular}

All values are the mean $\pm \mathrm{SD}$ of three replicates.

Sensory scores were assessed on a 5 point scale based on $1=$ extremely bad, $5=$ extremely good.

${ }^{\mathrm{A}, \mathrm{B}}$ Means within columns with different superscripts are significantly different $(p<0.05)$.

${ }^{\mathrm{a}, \mathrm{b}}$ Means within row with different superscripts are significantly different $(p<0.05)$. 
Table 7. Changes in sensory scores of yogurt beverage fortified with trypsin hydrolysate

\begin{tabular}{cccccc}
\hline \hline \multirow{2}{*}{$\begin{array}{c}\text { Sensory } \\
\text { parameter }\end{array}$} & $\begin{array}{c}\text { Storage } \\
\text { period (d) }\end{array}$ & \multicolumn{4}{c}{ Hydrolysate conc. (mg/mL) } \\
\cline { 3 - 6 } Odor & 0 & $4.1 \pm 0.32$ & $4.1 \pm 0.57$ & $4.3 \pm 0.67$ & $4.1 \pm 0.57$ \\
& 3 & $4.0 \pm 0.67^{\mathrm{b}}$ & $4.2 \pm 0.63^{\mathrm{ab}}$ & $4.6 \pm 0.52^{\mathrm{a}}$ & $4.4 \pm 0.52^{\mathrm{ab}}$ \\
& 7 & $4.2 \pm 0.63$ & $4.2 \pm 0.63$ & $4.4 \pm 0.70$ & $4.3 \pm 0.67$ \\
\hline \multirow{3}{*}{ Taste } & 0 & $4.1 \pm 0.32$ & $4.0 \pm 0.47$ & $4.3 \pm 0.67$ & $4.1 \pm 0.57$ \\
& 3 & $4.0 \pm 0.67^{\mathrm{b}}$ & $4.5 \pm 0.71^{\mathrm{ab}}$ & $4.6 \pm 0.52^{\mathrm{a}}$ & $4.4 \pm 0.52^{\mathrm{ab}}$ \\
& 7 & $4.3 \pm 0.67$ & $4.4 \pm 0.70$ & $4.5 \pm 0.71$ & $4.1 \pm 0.57$ \\
\hline \multirow{2}{*}{ Mouth } & 0 & $4.3 \pm 0.48$ & $4.1 \pm 0.57$ & $4.2 \pm 0.63$ & $4.2 \pm 0.63$ \\
feel & 3 & $3.9 \pm 0.57^{\mathrm{b}}$ & $4.4 \pm 0.52^{\mathrm{ab}}$ & $4.3 \pm 0.48^{\mathrm{ab}}$ & $4.5 \pm 0.53^{\mathrm{a}}$ \\
& 7 & $4.1 \pm 0.57$ & $4.4 \pm 0.70$ & $4.4 \pm 0.70$ & $4.0 \pm 0.47$ \\
\hline \multirow{3}{*}{ Color } & 0 & $4.2 \pm 0.32$ & $4.0 \pm 0.47$ & $4.0 \pm 0.47^{\mathrm{B}}$ & $4.3 \pm 0.67$ \\
& 3 & $4.0 \pm 0.67^{\mathrm{b}}$ & $4.5 \pm 0.53^{\mathrm{ab}}$ & $4.6 \pm 0.52^{\mathrm{Aa}}$ & $4.3 \pm 0.48^{\mathrm{ab}}$ \\
& 7 & $4.1 \pm 0.57$ & $4.4 \pm 0.70$ & $4.4 \pm 0.70^{\mathrm{AB}}$ & $4.1 \pm 0.57$ \\
\hline \multirow{2}{*}{ Total } & 0 & $4.1 \pm 0.57$ & $4.4 \pm 0.70$ & $4.0 \pm 0.47$ & $4.2 \pm 0.63$ \\
& 3 & $3.9 \pm 0.57^{\mathrm{b}}$ & $4.5 \pm 0.53^{\mathrm{a}}$ & $4.3 \pm 0.48^{\mathrm{ab}}$ & $4.4 \pm 0.48^{\mathrm{ab}}$ \\
& 7 & $4.2 \pm 0.63$ & $4.1 \pm 0.57$ & $4.1 \pm 0.57$ & $4.4 \pm 0.52$ \\
\hline
\end{tabular}

All values are the mean \pm SD of three replicates.

Sensory scores were assessed on a 5 point scale based on $1=$ extremely bad, $5=$ extremely good.

${ }^{\mathrm{A}, \mathrm{B}}$ Means within columns with different superscripts are significantly different $(p<0.05)$.

${ }^{a, b}$ Means within row with different superscripts are significantly different $(p<0.05)$.

ent in yogurt beverage fortified with alcalase hydrolysate. Also, the value of taste of $3 \mathrm{~d}$ and mouth feel of $3 \mathrm{~d}$ was significant different in yogurt beverage fortified with protamex hydrolysate. And total sensory evaluation was over 4.0 as "like level" in both cases.

The use of these hydrolysates in the manufacture of yogurt did not significantly influence $\mathrm{pH}$, titratable acidity, colority, LAB, or sensory evaluations during storage. Therefore, as stated above, the physicochemical characteristics of the hydrolysates are quite adequate for inclusion in yogurt beverage, as no unfavorable changes in yogurt beverage fortified with hydrolysates were observed. Our results demonstrate that whey protein hydrolysates have potential as an ingredient in the production of antihypertensive functional dairy product.

\section{Acknowledgements}

This study was supported by Korea Institute of Planning \& Evaluation for Technology of Food, Agriculture, Forestry \& Fisheries, Ministry for Food, Agriculture, Forestry and Fisheries Korea (Code \# 109136-3) and Priority Research Centers Program through the National Research Foundation of Korea (NRF) funded by the Ministry of
Education, Science, and Technology (2009-0093824).

\section{References}

1. AOAC (1995) Official methods of analysis. 16th ed, Association of Official Analytical Chemists, Washington, DC, USA.

2. Ariyoshi, Y. (1993) Angiotensin-converting enzyme inhibitor derived from food protein. Trends Food Sci. Technol. 4, 139-144.

3. Campbell, D. J. (2003) The renin-angiotensin and the kallikrein-kinin systems. Int. J. Biochem. Cell Biotechnol. 35, 784-791.

4. Christensen, J. E., Dudley, E. G., Pederson, J. A., and Steele, J. L. (1999) Peptidases and amino acid catabolism in lactic acid bacteria. Antonie van Leeuwenhoek 76, 217-246.

5. Clare, A. and Swaisgood, H. E. (2000) Bioactive milk peptides: a prospectus. J. Dairy Sci. 86, 1187-1195.

6. Cushman, D. W. and Cheung, H. S. (1971) Spectrophotometric assay and properties of the angiotensin-converting enzyme of rabbit lung. Biochem. Pharmacol. 20, 1637-1648.

7. Donkor, O. N., Henriksson, A., Vasiljevic, T., and Shah, N. P. (2007) Proteolytic activity of dairy lactic acid bacteria and probiotics as determinant of viability and in vitro angiotensin-converting enzyme inhibitory activity in fermented milk. Lait 87, 21-38.

8. FitzGerald, R. J. and Murray, B. A. (2006) Bioactive peptides and lactic fermentations. Int. J. Dairy Technol. 59, 118125.

9. Fuglsang, A., Nilsson, D., and Nyborg, N. C. B. (2002) Cardiovascular effects of fermented milk containing angiotensin-converting enzyme inhibitors evaluated in permanently catheterized, spontaneously hypertensive rats. Appl. Environ. Microbiol. 68, 3566-3569.

10. Fujita, H., Yamagami, T., and Ohshima, K. (2001) Effects of an ACE inhibitory agent, katsuobushi oligopeptide, in the spontaneously hypertensive rat and in borderline and mildly hypertensive subjects. Nutr. Res. 21, 1149-1158.

11. Jang, A. and Lee, M. (2005) Purification and identification of angiotensin converting enzyme inhibitory peptides from beef hydrolysates. Meat Sci. 69, 653-661.

12. Kim, S. B., Seo, I. S., Khan, M. A., Ki, K. S., Nam, M. S., and Kim, H. S. (2007) Separation of iron-binding protein from whey through enzymatic hydrolysis. Int. Dairy J. 17, 625-631.

13. Korhonen, H. J. T. and Pihlanto, A. (2006) Bioactive peptides: Production and functionality. Int. Dairy J. 16, 945-960.

14. Kunji, E. R. S., Mierau, I., Hagting, A., Poolman, B., and Konings, W. N. (1996) The proteolytic systems of lactic acid bacteria. Antonie van Leeuwenhoek 70, 187-221.

15. López-Fandino, R., Otte J., and van Camp, J. (2006) Physiological, chemical and technological aspects of milk-proteinderived peptides with antihypertensive and ACE-inhibitory activity. Int. Dairy J. 16, 1277-1293.

16. Minervini, F., Algaron, F., Rizzello, C. G., Fox, P. F., Monnet, V., and Gobbetti, M. (2003) Angiotensin I-converting- 
enzyme-inhibitory and antibacterial peptides from Lactobacillus helveticus PR4 proteinase-hydrolyzed caseins of milk from six species. Appl. Environ. Microbiol. 69, 5297-5305.

17. Nicholas, J. A. and Eric, C. R. (1996) Characterization of casein phosphopeptides prepared using alcalase. Enzyme Microb. Technol. 19, 202-207.

18. Otte, J., Qvist, K. B., Pedersen, M., Vogensen, F. K., and Flambard, B. (2003) ACE-inhibitory activity of fermented milks: Effects of culture and protein concentration. Poster at the Conference Milk Proteins: Quality and Health Aspects., Wadahl, Norway, pp. 13-16.

19. Pihlanto-Leppala, A., Koskinen, P., Piilola, K., Tupasela, T., and Korhonen, H. (2000) Angiotensin I-converting enzyme inhibitory properties of whey protein digests: Concentration and characterization of active peptides. J. Dairy Res. 67, 5364.

20. SAS (1999) SAS/STAT Software for PC. SAS Institute Inc., Cary, NC, USA.

21. Seppo, L., Jauhiainen, T., Poussa, T., and Korpela, R. (2003) A fermented milk high in bioactive peptides has a blood pressure-lowering effect in hypertensive subjects. Am. J. Clin. Nutr. 77, 326-330.

22. Shah, N. P. (2000) Effect of milk-derived bioactives: An overview. Br. J. Nutr. 84, S3-S10.

23. Silvia, S. V. and Malcata, F. X. (2005) Caseins as source of bioactive peptides. Int. Dairy J. 15, 1-15.

24. Sinha, R., Radha, C., Prakash, J., and Kaul, P. (2007) Whey protein hydrolysate: Functional properties, nutritional quality and utilization in beverage formulation. Food Chem. 101, 1484-1491.

25. Van der Ven, C., Grupen, H., Bont, D. B. A., and Voragen, A. G. J. (2002) Optimization of the angiotensin converting enzyme inhibition by whey protein hydrolysates using response surface methodology. Int. Dairy J. 12, 813-820.

26. Vermeirssen, V., Van Camp, J., Devos, L., and Verstraete, W. (2003) Release of angiotensin I converting enzyme (ACE) inhibitory activity during in vitro gastrointestinal digestion: From batch experiment to semicontinuous model. J. Agri. Food Chem. 51, 5680-5687.

27. Yamamoto, N. (1997) Antihypertensive peptides derived from food proteins. Biopolymers 43, 129-134.

28. Yamamoto, N., Akino, A., and Takano, T. (1994) Antihypertensive effect of the peptides derived from casein by an extracellular proteinase from Lactobacillus helveticus CP790. J. Dairy Sci. 77, 917-922.

29. Yusuf, S., Lonn, E., Bosch, J., and Gerstein, H. (1999) Summary of randomised trials of angiotensin converting enzyme inhibitors. Clin. Exp. Hypertens. 21, 835-845.

(Received 2011.10.27/Revised 2011.12.9/Accepted 2011.12.12) 